\title{
Variation diminishing-type properties for multivariate sampling Kantorovich operators
}

\author{
Laura Angeloni ${ }^{1}$ - Danilo Costarelli ${ }^{1}$ (D) Marco Seracini ${ }^{1} \cdot$ Gianluca Vinti $^{1}$. \\ Luca Zampogni ${ }^{1}$
}

Received: 25 June 2020 / Accepted: 13 August 2020 / Published online: 27 August 2020

(c) The Author(s) 2020

\begin{abstract}
In this paper we establish a variation-diminishing type estimate for the multivariate Kantorovich sampling operators with respect to the concept of multidimensional variation introduced by Tonelli. A sharper estimate can be achieved when step functions with compact support (digital images) are considered. Several examples of kernels have been presented.
\end{abstract}

Keywords Multivariate generalized sampling Kantorovich series · Variation-diminishing type property $\cdot$ Averaged type kernel $\cdot$ Smoothing in digital image processing $\cdot$ Product kernel

Mathematics Subject Classification $41 \mathrm{~A} 30 \cdot 41 \mathrm{~A} 05$

\section{Introduction}

The concept of variation and also of multidimensional variation of the type introduced by Tonelli, was implicitly one of the first topics addressed by Prof. Domenico Candeloro (Mimmo, for friends). He did so in his first two papers [20,21] where he studied the Geöcze area as a Burkill-Cesari integral and the link between the absolute continuity and the BurkillCesari integral, respectively. Subsequently, in the last years he has worked for a long time on the variational integrals in the sense of Henstock-Kurzweil; in particular, in his papers

To Mimmo, the Mentor, the Scientist and the Man, with deep esteem, gratitude, friendship and affection

The authors are partially supported by the "Department of Mathematics and Computer Science" of the University of Perugia (Italy) and are members of the "Research Italian Network on Approximation". The first, the fourth and and the fifth author have been partially supported within the projects "Metodi di Teoria dell'Approssimazione, Analisi Reale, Analisi Nonlineare e loro applicazioni” and "Integrazione,

Approssimazione, Analisi Nonlineare e loro Applicazioni", funded by the 2018 and 2019 basic research fund of the University of Perugia. Finally, the first and the second author of the paper have been partially supported within the 2020 GNAMPA-INdAM Project: "Metodi di analisi reale e di teoria della misura per l'approssimazione attraverso operatori discreti e applicazioni".

$\bigotimes \quad$ Danilo Costarelli

danilo.costarelli@unipg.it

Extended author information available on the last page of the article 
$[22,23]$, he managed to obtain, together with his co-authors, the existence of integrable selections in the variational sense, which was an open problem in Measure Theory for several years. It therefore seemed natural to us to dedicate the results of this paper to Mimmo, considering that these issues represented part of his scientific training in the early years and beyond. Each of us, in a different form, owes much to Mimmo; but surely together, we can express our gratitude for what he has left us, both from a scientific and a human point of view, and this contribution wants to be one of the many ways to tell him ... Thanks!

Working with families of operators in BV-spaces, a classical and important result that is usually investigated is an estimate of the variation of the operators in terms of the variation of the function to which they are applied: such property, known as "variation diminishing type estimate", can be obtained for several families of operators, well-known and used in approximation theory, such as the Bernstein polynomials, the convolution operators, the Mellin operators, the sampling operators and others (see, e.g., [3-5,9,14,32]). In this paper we study the case of multidimensional sampling Kantorovich operators, using the variation in the sense of Tonelli. We also consider separately the one-dimensional case (Theorem 2), that can be derived easily by the analogous result for the generalized sampling series [5]: in this case, for non-negative kernels, it is possible to obtain a classical variation diminishing result, i.e., the variation of the operators is smaller than the variation of the function itself. The multidimensional case (Theorem 1) is much more delicate: this is due to the "structure" of the Tonelli variation that is responsible, in particular, of the dependance of the constant in the estimate on the dimension of the space (this phenomenon is known as the curse-ofdimensionality occurring in several approximation problems), and to the particular form of the sampling Kantorovich operators. Nevertheless, the approximation properties of such family of operators, in their multidimensional form, are interesting and they have been widely studied in last years in view of their connections to Sampling Theory and Digital Image Processing, see e.g., [16,25,26,28-30,33,38]. Their definition has been introduced in 2007 in the univariate form [15] and subsequently it was extended to the multidimensional setting in [27]. The latter generalization has been given in order to have a class of approximation operators of the sampling-type, suitable in order to reconstruct not necessarily continuous signals, that is exactly the context in which the problem of processing (reconstruction, enhancement, smoothing etc) digital images is included [7,27].

In this direction, variation diminishing type estimates may have an applicative interpretation. Indeed, in case of step-type functions, that is, the mathematical model of digital images, it is possible to obtain a sharper estimate (Corollary 1 ) proving that the $L^{1}$-norm of the variation of the sections of the sampling Kantorovich operators, in case of non-negative kernels, is smaller than the $L^{1}$-norm of the variation of the sections of the function itself: from the applicative point of view, this produces a smoothing effect on the image reconstructed by means of the multidimensional sampling Kantorovich operators, with respect to the original one.

\section{Notations and preliminaries}

In the present paper, we denote by $B V(\mathbb{R}):=\left\{f: \mathbb{R} \longrightarrow \mathbb{R}: V_{\mathbb{R}}[f]<+\infty\right\}$ the space of functions of bounded variation on $\mathbb{R}$; the functional $V_{\mathbb{R}}[f]:=\sup _{[a, b] \subset \mathbb{R}} V_{[a, b]}[f]$ denotes the Jordan variation of $f$ over $\mathbb{R}$, and $V_{[a, b]}[f]=\sup \sum_{i=1}^{n}\left|f\left(x_{i}\right)-f\left(x_{i-1}\right)\right|$, where the supremum is taken over all the possible partitions $a=x_{0}<x_{1}<\ldots<x_{n}=b$ of the interval $[a, b]$, is the Jordan variation of $f$ over $[a, b][3,11,12]$. For what concerns the 
multivariate extension of the above concepts, several possible approaches can be found in the literature (see, e.g., [13]).

In particular, here we consider the concept of variation introduced by Tonelli [35] for two variables, extended to the general case of $\mathbb{R}^{N}$ by Radó and Vinti [34,37]. In order to recall it, we first introduce the following notation.

For a function $f: \mathbb{R}^{N} \rightarrow \mathbb{R}$ and $\mathrm{x}=\left(x_{1}, \ldots, x_{N}\right) \in \mathbb{R}^{N}$, we put $\mathrm{x}_{j}^{\prime}=$ $\left(x_{1}, \ldots, x_{j-1}, x_{j+1}, \ldots, x_{N}\right) \in \mathbb{R}^{N-1}, \mathrm{x}=\left(\mathrm{x}_{j}^{\prime}, x_{j}\right)$ and $f(\mathrm{x})=f\left(\mathrm{x}_{j}^{\prime}, x_{j}\right), j=1, \ldots, N$. Moreover, given an $N$-dimensional interval $I=\prod_{i=1}^{N}\left[a_{i}, b_{i}\right]$, by $I_{j}^{\prime}=\left[\mathrm{a}_{j}^{\prime}, \mathrm{b}_{j}^{\prime}\right]$ we will denote the $(N-1)$-dimensional interval obtained deleting by $I$ the $j$-th coordinate, i.e., $I=\left[\mathrm{a}_{j}^{\prime}, \mathrm{b}_{j}^{\prime}\right] \times\left[a_{j}, b_{j}\right], \quad j=1, \ldots, N$. Given a vector $\mathrm{x} \in \mathbb{R}^{N}$ and $\alpha \in \mathbb{R}$, we will use the usual notation for products and quotients, i.e., $\alpha \mathrm{x}=\left(\alpha x_{1}, \ldots, \alpha x_{N}\right)$ and, for $\alpha \neq 0$, $\frac{\mathrm{x}}{\alpha}=\left(\frac{x_{1}}{\alpha}, \ldots, \frac{x_{N}}{\alpha}\right)$. For more details and results about $B V$-spaces, see, e.g., $[2-4,8-11,13,31]$.

Now, we are able to recall the definition of the multivariate Tonelli variation. First of all, for any $I=\prod_{i=1}^{N}\left[a_{i}, b_{i}\right]$ and $j=1, \ldots, N$ we consider the $(N-1)$-dimensional integrals (Tonelli integrals)

$$
\Phi_{j}(f, I):=\int_{\left[\mathrm{a}_{j}^{\prime}, \mathrm{b}_{j}^{\prime}\right]} V_{\left[a_{j}, b_{j}\right]}\left[f\left(\mathrm{x}_{j}^{\prime}, \cdot\right)\right] d \mathrm{x}_{j}^{\prime},
$$

$\left(V_{\left[a_{j}, b_{j}\right]}\left[f\left(\mathrm{x}_{j}^{\prime}, \cdot\right)\right]\right.$ is the one-dimensional Jordan variation of the $j-t h$ section of $\left.f\right)$ and their Euclidean norm $\Phi(f, I):=\left\{\sum_{j=1}^{N} \Phi_{j}^{2}(f, I)\right\}^{\frac{1}{2}}$. Note that $\Phi(f, I)=+\infty$ if $\Phi_{j}(f, I)=$ $+\infty$ for some $j=1, \ldots, N$.

Then the variation of $f$ on $I \subset \mathbb{R}^{N}$ can be defined as

$$
V_{I}[f]:=\sup \sum_{k=1}^{m} \Phi\left(f, J_{k}\right),
$$

where the supremum is taken over all the finite families of $N$-dimensional intervals $\left\{J_{1}, \ldots, J_{m}\right\}$ which form partitions of $I$. Passing to the supremum over all the intervals $I \subset \mathbb{R}^{N}$, we obtain the variation of $f$ over the whole $\mathbb{R}^{N}$, i.e.,

$$
V[f]:=\sup _{I \subset \mathbb{R}^{N}} V_{I}[f] .
$$

Definition 1 A measurable and bounded function $f: \mathbb{R}^{N} \rightarrow \mathbb{R}$ is said to be of bounded variation $\left(f \in B V\left(\mathbb{R}^{N}\right)\right)$ if $V[f]<+\infty$.

By the definition it immediately follows that, for every $f \in B V\left(\mathbb{R}^{N}\right), \nabla f$ exists a.e. in $\mathbb{R}^{N}$ and $\frac{\partial f}{\partial x_{j}} \in L^{1}\left(\mathbb{R}^{N}\right)$, for every $j=1, \ldots, N$ (see e.g. [34,37]).

Definition 2 A function $f: \mathbb{R}^{N} \rightarrow \mathbb{R}$ is locally absolutely continuous in the sense of Tonelli $\left(f \in A C_{l o c}\left(\mathbb{R}^{N}\right)\right)$ if, for every interval $I=\prod_{i=1}^{N}\left[a_{i}, b_{i}\right]$ and for every $j=1,2, \ldots, N$, the $j$-th section of $f, f\left(\mathrm{x}_{j}^{\prime}, \cdot\right):\left[a_{j}, b_{j}\right] \rightarrow \mathbb{R}$ is absolutely continuous for almost every $\mathrm{x}_{j}^{\prime} \in\left[\mathrm{a}_{j}^{\prime}, \mathrm{b}_{j}^{\prime}\right]$.

Denoting by $A C\left(\mathbb{R}^{N}\right):=B V\left(\mathbb{R}^{N}\right) \cap A C_{\text {loc }}\left(\mathbb{R}^{N}\right)$, it is well known that, if $f \in A C\left(\mathbb{R}^{N}\right)$ then $V[f]=\int_{\mathbb{R}^{N}}|\nabla f(\mathrm{x})| d \mathrm{x}($ see $[12,31,34,37])$.

In order to provide some estimates with respect to the multivariate Tonelli variation for a family of multivariate sampling-type operators, we now introduce the following definition.

A function $\chi: \mathbb{R}^{N} \rightarrow \mathbb{R}$ will be called a kernel if it satisfies the following assumptions: 
$\left(\chi_{1}\right) \quad \chi \in L^{1}\left(\mathbb{R}^{N}\right)$ is such that $\sum_{\mathrm{k} \in \mathbb{Z}^{N}} \chi(\mathrm{u}-\mathrm{k})=1$, for every $\mathrm{u} \in \mathbb{R}^{N}$;

$\left(\chi_{2}\right) \quad A_{\chi}:=\sup _{\mathrm{u} \in \mathbb{R}^{N}} \sum_{\mathrm{k} \in \mathbb{Z}^{N}}|\chi(\mathrm{u}-\mathrm{k})|<+\infty$, where the convergence of the series is uniform on the compact sets of $\mathbb{R}^{N}$.

In particular, the above conditions are usually satisfied by the multivariate discrete approximate identities (see, e.g., [19]).

This paper deals with the family of multivariate sampling Kantorovich operators [27], defined as

$$
\left(K_{w} f\right)(\mathrm{t}):=\sum_{\mathrm{k} \in \mathbb{Z}^{N}}\left[w^{N} \int_{\left[\frac{\mathrm{k}}{w}, \frac{\mathrm{k}+1}{w}\right]} f(\mathrm{u}) d \mathrm{u}\right] \chi(w \mathrm{t}-\mathrm{k}), \quad \mathrm{t} \in \mathbb{R}^{N}, w>0 .
$$

The sampling Kantorovich operators represent the $L^{1}$-version of the generalized sampling series

$$
\left(S_{w} f\right)(\mathrm{t}):=\sum_{\mathrm{k} \in \mathbb{Z}^{N}} f\left(\frac{\mathrm{k}}{w}\right) \chi(w \mathrm{t}-\mathrm{k}), \quad t \in \mathbb{R}^{N}, w>0,
$$

(see, e.g., $[17,18])$.

Note that the operators $\left(K_{w} f\right)_{w>0}$ and $\left(S_{w} f\right)_{w>0}$ are well-defined, for instance, for any $f \in B V\left(\mathbb{R}^{N}\right)$ : indeed in this case it turns out that $|f(\mathrm{t})| \leq M$, for some $M>0$, and thus, by $\left(\chi_{2}\right),\left|\left(K_{w} f\right)(\mathrm{t})\right| \leq M \sum_{\mathrm{k} \in \mathbb{Z}^{N}} \chi(w \mathrm{t}-\mathrm{k}) \leq M A_{\chi}, \mathrm{t} \in \mathbb{R}^{N}$.

In the present paper, in order to establish some estimates with respect to the Tonelli variation for the operators $K_{w}$, we consider kernels which are given by the product of onedimensional kernels of averaged form (see, e.g., [5,7,32]). More precisely, we define

$$
\bar{\chi}_{m}(\mathrm{t}):=\prod_{i=1}^{N} \bar{\chi}_{i, m}\left(t_{i}\right)
$$

where

$$
\bar{\chi}_{i, m}(t):=\frac{1}{m} \int_{-\frac{m}{2}}^{\frac{m}{2}} \chi_{i}(t+v) d v
$$

for some $m \in \mathbb{N}$, and $\chi_{i}: \mathbb{R} \longrightarrow \mathbb{R}$ is a (one-dimensional) kernel for every $i=1, \ldots, N$ (i.e., satisfying $\left(\chi_{1}\right)$ and $\left(\chi_{2}\right)$ with $\left.N=1\right)$. It is easy to see that $\bar{\chi}_{m}$ is a kernel itself and that, for every $i=1, \ldots, N$,

$$
\left\|\bar{\chi}_{i, m}\right\|_{1} \leq\left\|\chi_{i}\right\|_{1} .
$$

Moreover, $\bar{\chi}_{m}$ is everywhere differentiable and

$$
\frac{\partial \bar{\chi}_{m}}{\partial t_{j}}(\mathrm{t})=\frac{1}{m} \prod_{i \neq j} \bar{\chi}_{i, m}\left(t_{i}\right)\left[\chi_{j}\left(t_{j}+\frac{m}{2}\right)-\chi_{j}\left(t_{j}-\frac{m}{2}\right)\right], \quad t \in \mathbb{R}^{N} .
$$

From now on, for the sake of simplicity, we will denote by $\bar{K}_{w}^{m}$ and $\bar{S}_{w}^{m}$ the multivariate Kantorovich and generalized sampling series, respectively, both based upon the averaged product kernel $\bar{\chi}_{m}$.

\section{The main results}

In this section, we prove the main result of the paper, i.e., an estimate for the variation of the multivariate sampling Kantorovich operators. 
Theorem 1 For any $f \in B V\left(\mathbb{R}^{N}\right), m \in \mathbb{N}, w>0$,

$$
V\left[\bar{K}_{w}^{m} f\right] \leq N \frac{m+1}{m} \prod_{i=1}^{N}\left\|\chi_{i}\right\|_{1} V[f],
$$

and hence $\bar{K}_{w}^{m} f \in B V\left(\mathbb{R}^{N}\right)$. Moreover $\bar{K}_{w}^{m} f \in A C\left(\mathbb{R}^{N}\right)$.

Proof First of all we can observe that, for $f \in B V\left(\mathbb{R}^{N}\right), \frac{\partial \bar{K}_{w}^{m} f}{\partial t_{j}}(\mathrm{t})$ exists for every $t \in \mathbb{R}^{N}$. Indeed, for every $j=1, \ldots, N, t \in \mathbb{R}^{N}$, there holds

$$
\begin{aligned}
\frac{\partial \bar{K}_{w}^{m} f}{\partial t_{j}}(\mathrm{t})= & \frac{w^{N+1}}{m} \sum_{\mathrm{k} \in \mathbb{Z}^{N}}\left[\int_{\left[\frac{\mathrm{k}}{w}, \frac{\mathrm{k}+1}{w}\right]} f(\mathrm{u}) d \mathrm{u}\right] \prod_{i \neq j} \bar{\chi}_{i, m}\left(w t_{i}-k_{i}\right)\left[\chi_{j}\left(w t_{j}-k_{j}+\frac{m}{2}\right)\right. \\
& \left.-\chi_{j}\left(w t_{j}-k_{j}-\frac{m}{2}\right)\right],
\end{aligned}
$$

and hence, using $\left(\chi_{2}\right)$ for each one-dimensional kernel $\bar{\chi}_{i, m}, \chi_{j}$, we have, for every $t \in \mathbb{R}^{N}$,

$$
\begin{aligned}
\left|\frac{\partial \bar{K}_{w}^{m} f}{\partial t_{j}}(\mathrm{t})\right| \leq & \frac{w}{m} M \sum_{k \in \mathbb{Z}^{N}} \prod_{i \neq j}\left|\bar{\chi}_{i, m}\left(w t_{i}-k_{i}\right)\right|\left[\left|\chi_{j}\left(w t_{j}-k_{j}+\frac{m}{2}\right)\right|\right. \\
& \left.+\left|\chi_{j}\left(w t_{j}-k_{j}-\frac{m}{2}\right)\right|\right] \\
\leq & \frac{2 w}{m} M \prod_{i \neq j} A_{\bar{\chi}_{i, m}} A_{\chi_{j}} \leq \frac{2 w}{m} M \prod_{i=1}^{N} A_{\chi_{i}}<+\infty
\end{aligned}
$$

The estimate (3) proves that $\bar{K}_{w}^{m} f \in A C_{l o c}\left(\mathbb{R}^{N}\right)$. Now, using (2) and the change of variable $\widetilde{k}_{j}=k_{j}+m$, and $\widetilde{k}_{i}=k_{i}$, if $i \neq j$, we can write what follows

$$
\begin{aligned}
\frac{\partial \bar{K}_{w}^{m} f}{\partial t_{j}}(\mathrm{t})= & \frac{w^{N+1}}{m} \sum_{\mathrm{k} \in \mathbb{Z}^{N}}\left[\int_{\left[\frac{\mathrm{k}}{w}, \frac{\mathrm{k}+1}{w}\right]} f(\mathrm{u}) d \mathrm{u}\right] \\
& \times\left(\prod_{i \neq j} \bar{\chi}_{i, m}\left(w t_{i}-k_{i}\right)\right) \chi_{j}\left(w t_{j}-k_{j}+\frac{m}{2}\right) \\
& -\frac{w^{N+1}}{m} \sum_{\widetilde{\mathrm{k}} \in \mathbb{Z}^{N}}\left[\int_{\prod_{i \neq j}\left[\frac{\widetilde{k}_{i}}{w}, \frac{\widetilde{k}_{i}+1}{w}\right] \times\left[\frac{\widetilde{k}_{j}-m}{w}, \frac{\widetilde{k}_{j}-m+1}{w}\right]} f(\mathrm{u}) d \mathrm{u}\right] \\
& \times\left(\prod_{i \neq j} \bar{\chi}_{i, m}\left(w t_{i}-\widetilde{k}_{i}\right)\right) \chi_{j}\left(w t_{j}-\widetilde{k}_{j}+\frac{m}{2}\right) \\
= & \frac{w^{N+1}}{m} \sum_{\mathrm{k} \in \mathbb{Z}^{N}}\left[\int_{\left[\frac{\mathrm{k}}{w}, \frac{\mathrm{k}+1}{w}\right]} f(\mathrm{u}) d \mathrm{u}-\int\left[\frac{\mathrm{k}_{j}^{\prime}}{w}, \frac{\mathrm{k}_{j}^{\prime}+1}{w}\right] \times\left[\frac{k_{j}-m}{w}, \frac{k_{j}-m+1}{w}\right]\right. \\
& \times\left(\prod_{i \neq j} \bar{\chi}_{i, m}\left(w t_{i}-k_{i}\right)\right) \chi_{j}\left(w t_{j}-k_{j}+\frac{m}{2}\right) .
\end{aligned}
$$

Now, putting $v_{j}=u_{j}+m / w$, and $v_{i}=u_{i}$, if $i \neq j$, in the second integral, we get 


$$
\begin{aligned}
& \left|\frac{\partial \bar{K}_{w}^{m} f}{\partial t_{j}}(\mathrm{t})\right| \leq \frac{w^{N+1}}{m} \\
& \times \sum_{\mathrm{k} \in \mathbb{Z}^{N}}\left|\int_{\left[\frac{k}{w}, \frac{k+1}{w}\right]} f(\mathrm{u}) d \mathrm{u}-\int_{\left[\frac{k_{j}^{\prime}}{w}, \frac{k_{j}^{\prime}+1}{w}\right] \times\left[\frac{k_{j}}{w}, \frac{k_{j}+1}{w}\right]} f\left(\mathrm{v}_{j}^{\prime}, v_{j}-\frac{m}{w}\right) d \mathrm{v}\left(\prod_{i \neq j}\left|\bar{\chi}_{i, m}\left(w t_{i}-k_{i}\right)\right|\right)\right| \chi_{j}\left(w t_{j}-k_{j}+\frac{m}{2}\right) \mid \\
& \leq \frac{w^{N+1}}{m} \sum_{\mathrm{k} \in \mathbb{Z}^{N}} \int_{\left[\frac{\mathrm{k}}{w}, \frac{k+1}{w}\right]}\left|f(\mathrm{u})-f\left(\mathrm{u}_{j}^{\prime}, u_{j}-\frac{m}{w}\right)\right| d \mathrm{u}\left(\prod_{i \neq j}\left|\bar{\chi}_{i, m}\left(w t_{i}-k_{i}\right)\right|\right)\left|\chi_{j}\left(w t_{j}-k_{j}+\frac{m}{2}\right)\right| \\
& \leq \frac{w^{N+1}}{m} \sum_{\mathrm{k} \in \mathbb{Z}^{N}} \int_{\left[\frac{k_{j}^{\prime}}{w}, \frac{k_{j}^{\prime}+1}{w}\right]}\left[\int_{\left(k_{j} / w\right)}^{\left(k_{j}+1\right) / w} V_{\left[\frac{k_{j}-m}{w}, \frac{k_{j}+1}{w}\right]}\left[f\left(\mathrm{u}_{j}^{\prime}, \cdot\right)\right] d \mathrm{u}\left(\prod_{i \neq j}\left|\bar{\chi}_{i, m}\left(w t_{i}-k_{i}\right)\right|\right)\left|\chi_{j}\left(w t_{j}-k_{j}+\frac{m}{2}\right)\right|\right. \\
& \frac{w^{N}}{m} \sum_{k \in \mathbb{Z}^{\mathbb{N}}}\left(\prod_{i \neq j}\left|\bar{\chi}_{i, m}\left(w t_{i}-k_{i}\right)\right|\right) \int_{\left[\frac{k_{j}^{\prime}}{w}, \frac{k_{j}^{\prime}+1}{w}\right]}\left[V_{\left[\frac{k_{j}-m}{w}, \frac{k_{j}-m+1}{w}\right]}\left[f\left(u_{j}^{\prime}, \cdot\right)\right]+\ldots+V_{\left[\frac{k_{j}}{w}, \frac{k_{j}+1}{w}\right]}\left[f\left(u_{j}^{\prime}, \cdot\right)\right]\right] d u_{j}^{\prime} \\
& \times\left|\chi_{j}\left(w t_{j}-k_{j}+\frac{m}{2}\right)\right| .
\end{aligned}
$$

Now, using the Fubini-Tonelli theorem, the change of variable $y_{j}=w t_{j}-k_{j}+m / 2$, $y_{i}=w t_{i}-k_{i}, i \neq j$, and the inequality (1) we have

$$
\begin{aligned}
V\left[\bar{K}_{w}^{m} f\right]= & \int_{\mathbb{R}^{N}}\left(\sum_{j=1}^{N}\left|\frac{\partial \bar{K}_{w}^{m} f}{\partial t_{j}}(\mathrm{t})\right|^{2}\right)^{1 / 2} d \mathrm{t} \leq \sum_{j=1}^{N} \int_{\mathbb{R}^{N}}\left|\frac{\partial \bar{K}_{w}^{m} f}{\partial t_{j}}(\mathrm{t})\right| d \mathrm{t} \\
\leq & \frac{w^{N}}{m} \sum_{j=1}^{N} \sum_{\mathrm{k} \in \mathbb{Z}^{N}} \int_{\left[\frac{k_{j}^{\prime}}{w}, \frac{k_{j}^{\prime}+1}{w}\right]}\left[V_{\left[\frac{k_{j}-m}{w}, \frac{k_{j}-m+1}{w}\right]}\left[f\left(\mathrm{u}_{j}^{\prime}, \cdot\right)\right]+\ldots+V_{\left[\frac{k_{j}}{w}, \frac{k_{j}+1}{w}\right]}\left[f\left(\mathrm{u}_{j}^{\prime}, \cdot\right)\right]\right] d \mathrm{u}_{j}^{\prime} \\
& \times \int_{\mathbb{R}^{N}}\left(\prod_{i \neq j}\left|\bar{\chi}_{i, m}\left(w t_{i}-k_{i}\right)\right|\right)\left|\chi_{j}\left(w t_{j}-k_{j}+\frac{m}{2}\right)\right| d \mathrm{t} \\
\leq & \frac{1}{m} \sum_{j=1}^{N} \sum_{\mathrm{k}_{j}^{\prime} \in \mathbb{Z}^{N-1}} \int_{\left[\frac{k_{j}^{\prime}}{w}, \frac{k_{j}^{\prime}+1}{w}\right]} \sum_{k_{j} \in \mathbb{Z}^{2}}\left[V_{\left[\frac{k_{j}-m}{w}, \frac{k_{j}-m+1}{w}\right]}\left[f\left(\mathrm{u}_{j}^{\prime}, \cdot\right)\right]+\ldots+V_{\left[\frac{k_{j}}{w}, \frac{k_{j}+1}{w}\right]}\left[f\left(\mathrm{u}_{j}^{\prime}, \cdot\right)\right]\right] d \mathrm{u}_{j}^{\prime} \\
& \times \int_{\mathbb{R}^{N}}\left|\chi_{j}\left(y_{j}\right)\right| \prod_{i \neq j}\left|\bar{\chi}_{i, m}\left(y_{i}\right)\right| d \mathrm{Y} \leq \frac{m+1}{m} \sum_{j=1}^{N} \prod_{i=1}^{N}\left\|\chi_{i}\right\|_{1} \sum_{\mathrm{k}_{j}^{\prime} \in \mathbb{Z}^{N-1}} \int_{\left[\frac{k_{j}^{\prime}}{w}, \frac{k_{j}^{\prime}+1}{w}\right]} V_{\mathbb{R}}\left[f\left(\mathrm{u}_{j}^{\prime}, \cdot\right)\right] d \mathrm{u}_{j}^{\prime} \\
= & \frac{m+1}{m} \prod_{i=1}^{N}\left\|\chi_{i}\right\|_{1} \sum_{j=1}^{N} \int_{\mathbb{R}^{N-1}} V_{\mathbb{R}}\left[f\left(\mathrm{u}_{j}^{\prime}, \cdot\right)\right] d \mathrm{u}_{j}^{\prime} .
\end{aligned}
$$

Now notice that, for $I=\prod_{i=1}^{N}\left[a_{i}, b_{i}\right], \Phi_{j}(f, I)=\int_{I_{j}^{\prime}} V_{\left[a_{j}, b_{j}\right]}\left[f\left(\mathrm{u}_{j}^{\prime}, \cdot\right)\right] d \mathrm{u}_{j}^{\prime} \leq$ $\Phi(f, I) \leq V_{I}[f], j=1, \ldots, N$, and so, passing to the supremum over $I \subset \mathbb{R}^{N}$, $\int_{\mathbb{R}^{N-1}} V_{\mathbb{R}}\left[f\left(\mathrm{u}_{j}^{\prime}, \cdot\right)\right] d \mathrm{u}_{j}^{\prime} \leq V[f]$; therefore

$$
V\left[\bar{K}_{w}^{m} f\right] \leq N \frac{m+1}{m} \prod_{i=1}^{N}\left\|\chi_{i}\right\|_{1} V[f] .
$$

Hence $\bar{K}_{w}^{m} f \in B V\left(\mathbb{R}^{N}\right)$ and therefore $\bar{K}_{w}^{m} f \in A C\left(\mathbb{R}^{N}\right)$.

In the one-dimensional case, namely for $\left(\bar{K}_{w}^{m} f\right)(t):=\sum_{k \in \mathbb{Z}} w\left(\int_{\frac{k}{w}}^{\frac{k+1}{w}} f(u) d u\right) \bar{\chi}_{m}(w t-$ $k), t \in \mathbb{R}, w>0$, where $\bar{\chi}_{m}(t)=1 / m \int_{-\frac{m}{2}}^{\frac{m}{2}} \chi(t+v) d v$ and $\chi$ is a one-dimensional kernel, it is possible to obtain a sharper estimate. Notice that, in case of a non-negative kernel, this gives a classical variation-diminishing result, since $\|\chi\|_{1}=1$. Namely, we may obtain

Theorem 2 For $f \in B V(\mathbb{R}), m \in \mathbb{N}, w>0$, there holds

$$
V\left[\bar{K}_{w}^{m} f\right] \leq\|\chi\|_{1} V[f] .
$$


Therefore $\bar{K}_{w}^{m} f \in B V(\mathbb{R})$ and so $\bar{K}_{w}^{m} f \in A C(\mathbb{R})$.

Proof It is easy to see that $\left(\bar{K}_{w}^{m} f\right)(t)=\left(\bar{S}_{w}^{m} I_{w}^{\varphi} f\right)(t), t \in \mathbb{R}$, where $\left(I_{w}^{\varphi} f\right)(t):=$ $w \int_{\mathbb{R}} f(u) \varphi(w(t-u)) d u, w>0$, for $\varphi(u)=\mathbf{1}_{[-1,0]}(u)$ (see [6]). Therefore, taking into account of Proposition 5.1 of [14] (with $D=\|w \varphi(w \cdot)\|_{1}=1$ ) and of Proposition 1 of [5] (notice that the constant that multiplies $V[f]$ is $\|\chi\|_{1}$ ), there holds

$$
V\left[\bar{K}_{w}^{m} f\right]=V\left[\bar{S}_{w}^{m} I_{w}^{\varphi} f\right] \leq\|\chi\|_{1} V\left[I_{w}^{\varphi} f\right] \leq\|\chi\|_{1} V[f],
$$

and so $\bar{K}_{w}^{m} f \in B V(\mathbb{R})$. Therefore $\bar{K}_{w}^{m} f \in A C(\mathbb{R})$ since, as in Theorem 1 , it can be proved that $\bar{K}_{w}^{m} f \in A C_{l o c}(\mathbb{R})$.

\section{Examples and applications}

In the present section, we discuss some applicative aspects of the theory of sampling Kantorovich operators, in particular in the present setting of BV-spaces. For this purpose, we first recall that some concrete applications of the above operators to the reconstruction and the enhancement of digital images have already been obtained in $[25,26]$.

Any given static gray scale image (matrix) $M=\left(m_{i, j}\right), i, j=0, \ldots, n-1$, can be easily represented (from the analytic point of view) by a function of two variables with compact support on the square $[0, n] \times[0, n]$. More precisely, we can define the so-called image function as follows

$$
I_{M}(x, y):=\sum_{i=0}^{n-1} \sum_{j=0}^{n-1} m_{i, j} \mathbf{1}_{i, j}(x, y), \quad(x, y) \in \mathbb{R}^{2},
$$

where $\mathbf{1}_{i, j}$ denotes the characteristic functions of the set $[i, i+1) \times[j, j+1)$.

Obviously any step function with compact support (as $I_{M}$ ) is in fact a function belonging to $B V\left(\mathbb{R}^{2}\right)$. In such particular case, besides the estimate established in Theorem 1 , that obviously holds, a sharper estimate for the variation of the two-dimensional sampling Kantorovich operators can be proved, in fact resulting a variation diminishing type property.

Corollary 1 Let $f: \mathbb{R}^{N} \rightarrow \mathbb{R}$ be a step function with compact support $[\mathrm{a}, \mathrm{b}] \subset \mathbb{R}^{N}$, where $\mathrm{a}, \mathrm{b} \in \mathbb{Z}^{N}, a_{i}<b_{i}, i=1, \ldots, N$, and $f(\mathrm{x})=f(\mathrm{i})$ for every $\mathrm{x} \in[\mathrm{i}, \mathrm{j}[$, i.e., $f$ is constant on each interval of a grid of multi-dimensional intervals of the form $[i, j] \subset[a, b]$, with $i, j \in \mathbb{Z}^{N}$, and $\left|j_{v}-i_{v}\right|=1, v=1, \ldots, N$, that form a partition of $[\mathrm{a}, \mathrm{b} \mathrm{b}$. Then, for every $w \in \mathbb{N}$ and $m \in \mathbb{N}$,

$$
\Phi_{j}\left(\bar{K}_{w}^{m} f,[\mathrm{a}, \mathrm{b}]\right) \leq \prod_{i=1}^{N}\left\|\chi_{i}\right\|_{1} \Phi_{j}\left(\bar{K}_{w}^{m} f,[\mathrm{a}, \mathrm{b}]\right), \quad j=1, \ldots, N,
$$

and therefore

$$
V\left[\bar{K}_{w}^{m} f\right] \leq N \prod_{i=1}^{N}\left\|\chi_{i}\right\|_{1} V[f] .
$$

In particular, if $\chi_{i}$ is non-negative, $i=1, \ldots, N$, it turns out that

$$
\Phi_{j}\left(\bar{K}_{w}^{m} f,[\mathrm{a}, \mathrm{b}]\right) \leq \Phi_{j}\left(\bar{K}_{w}^{m} f,[\mathrm{a}, \mathrm{b}]\right), j=1, \ldots, N, \text { and } V\left[\bar{K}_{w}^{m} f\right] \leq N V[f] .
$$



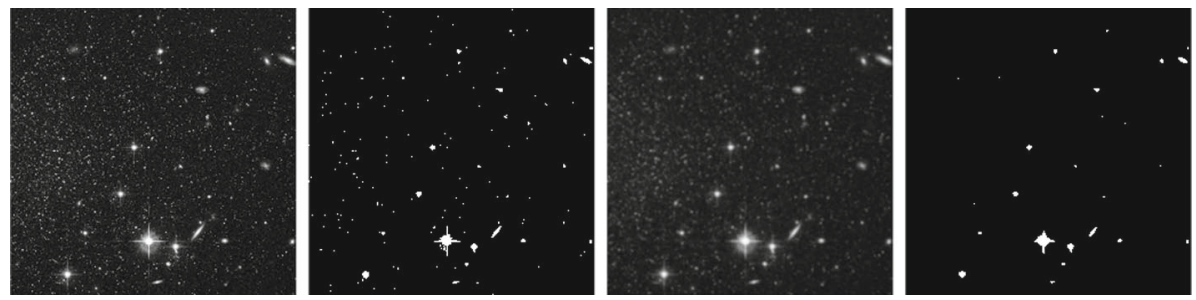

Fig. 1 a Original image; $\mathbf{b}$ result of thresholding on the original image; $\mathbf{c}$ reconstruction of the original image by means of the operator $K_{w}^{m}, w=5$, based upon the bivariate averaged Fejér kernel with $m=10$; $\mathbf{d}$ result of thresholding on the reconstructed image

Proof Observing that, for any step function $f$ as above and $w \in \mathbb{N}$ it turns out that $\bar{K}_{w}^{m} f=$ $\bar{S}_{w}^{m} f$, the proof immediately follows by Proposition 5 of [7].

As a consequence of (4), it turns out that for any image (matrix) $M$, the two-dimensional sampling Kantorovich operator $\bar{K}_{w}^{m} I_{M}, w, m \in \mathbb{N}$, based on $\bar{\chi}_{m}$ and generated by a nonnegative kernel $\chi$, allows to obtain a filtered image in which globally, the variations of the sections of the image are reduced: this may be interpreted as a reduction of the "jumps of gray levels" with respect to the original image $M$, producing a smoothing effect on the reconstructed image (see Fig. 1c). As it is well-known, smoothing procedures have a wide range of applications in digital image processing: among them, image enhancement, noise reduction, automatic selection procedures. Figure 1 illustrates an example of application of our operators to the latter problem. The aim is to produce an automatic procedure to select "big" objects from an image (the original, Fig. 1a, is a $250 \times 250$ Hubble space image): this can be done by filtering the image with the sampling Kantorovich operator, producing a smoothing effect (Fig. 1c), and hence applying thresholding to the filtered image (Fig. 1d). This produces an image where just the largest objects are detected. The importance of the application of the smoothing filter in this process can be easily deduced by comparing Fig. $1 \mathrm{~d}$ to the result of thresholding applied directly to the original image (Fig. 1b).

Now, we can furnish some examples of kernels for which the above results hold.

As a first example, we can consider the multivariate product kernel of the averaged type generated by the well-known Fejér kernel, defined by

$$
F(x):=\frac{1}{2} \operatorname{sinc}^{2}(x / 2), \quad x \in \mathbb{R},
$$

where the sinc-function (see, e.g., [6,24]) is of the form $\operatorname{sinc}(x):=\left\{\begin{array}{ll}\frac{\sin (\pi x)}{\pi x}, & x \neq 0, \\ 1, & x=0 .\end{array}\right.$ The one-dimensional averaged Fejér kernel is then defined as

$$
\bar{F}_{m}(t):=\frac{1}{2 m} \int_{-m / 2}^{m / 2} \operatorname{sinc}^{2}\left(\frac{t+v}{2}\right) d v, \quad t \in \mathbb{R}, \quad m \in \mathbb{N},
$$

and its corresponding multivariate version is $\mathcal{F}_{m}(\mathrm{t}):=\prod_{i=1}^{N} \bar{F}_{m}\left(t_{i}\right), t \in \mathbb{R}^{N}$. In practice, the averaged Fejér kernel represents the $L^{1}$-version of the well-known Lanczos kernel [32], which is defined as an averaged of a sinc-type function. Note that the Fejér kernel is nonnegative and then for the multivariate sampling Kantorovich series based upon $\mathcal{F}_{m}$ hold the variation diminishing type properties established in both Theorem 1 and Corollary 1. 
Other examples of one-dimensional non-negative kernels with unbounded support that can be used to define product averaged type kernels are, e.g., the Jackson-type kernels, defined by

$$
J_{n}(x):=c_{n} \operatorname{sinc}^{2 n}\left(\frac{x}{2 n \pi \alpha}\right), \quad x \in \mathbb{R},
$$

with $n \in \mathbb{N}, \alpha \geq 1$, and $c_{n}$ a non-zero normalization coefficient, given by $c_{n}:=$ $\left[\int_{\mathbb{R}} \operatorname{sinc}^{2 n}\left(\frac{u}{2 n \pi \alpha}\right) d u\right]^{-1}$, [33]. The averaged-kernels by means of the functions $J_{n}$ and their multivariate product can be constructed as in the case of Fejér kernel.

Now, in order to give examples of duration limited kernels, i.e., one-dimensional kernels with compact support, we recall the definition of the well-known central B-spline of order $n \in \mathbb{N}$ (see, e.g., $[1,36]$ ), defined by

$$
M_{n}(x):=\frac{1}{(n-1) !} \sum_{i=0}^{n}(-1)^{i}\left(\begin{array}{l}
n \\
i
\end{array}\right)\left(\frac{n}{2}+x-i\right)_{+}^{n-1}, \quad x \in \mathbb{R},
$$

where $(x)_{+}:=\max \{x, 0\}$ denotes "the positive part" of $x \in \mathbb{R}$.

Now, let us denote by

$$
\bar{M}_{n, m}(t):=m^{-1} \int_{-m / 2}^{m / 2} M_{n}(t+v) d v, \quad t \in \mathbb{R},
$$

the averaged B-spline kernel of order $n \in \mathbb{N}$. Recalling that $M_{n}^{\prime}(t)=M_{n-1}(t+1 / 2)-$ $M_{n-1}(t-1 / 2), t \in \mathbb{R}(n \geq 2)$, for $m=1$ we have $\bar{M}_{n, 1}^{\prime}(t)=M_{n}(t+1 / 2)-M_{n}(t-1 / 2)=$ $M_{n+1}^{\prime}(t), t \in \mathbb{R}(n \geq 1)$, from which we can obtain that $\bar{M}_{n, 1}(t)=M_{n+1}(t), \quad t \in \mathbb{R}$, for every $n \in \mathbb{N}$, namely, the averaged kernel with $m=1$ generated by a central B-spline of order $n$ is a B-spline itself of order $n+1$. Thus, the multivariate averaged type product kernel with $m=1$ and generated by $M_{n}$ is $\mathcal{M}_{1}^{n}(\mathrm{t}):=\prod_{i=1}^{N} \bar{M}_{n, 1}\left(t_{i}\right)=\prod_{i=1}^{N} M_{n+1}\left(t_{i}\right), \quad \mathrm{t} \in \mathbb{R}^{N}$. In practice, in the latter case the multivariate sampling Kantorovich series based upon the product of $N$ averaged B-spline $M_{n}$ with $m=1$ coincide with the sampling Kantorovich operators based upon the multidimensional central B-spline of order $n+1$.

Funding Open access funding provided by Universitá degli Studi di Perugia within the CRUI-CARE Agreement.

\section{Compliance with ethical standards}

Conflict of interest On behalf of all authors, the corresponding author states that there is no conflict of interest.

Open Access This article is licensed under a Creative Commons Attribution 4.0 International License, which permits use, sharing, adaptation, distribution and reproduction in any medium or format, as long as you give appropriate credit to the original author(s) and the source, provide a link to the Creative Commons licence, and indicate if changes were made. The images or other third party material in this article are included in the article's Creative Commons licence, unless indicated otherwise in a credit line to the material. If material is not included in the article's Creative Commons licence and your intended use is not permitted by statutory regulation or exceeds the permitted use, you will need to obtain permission directly from the copyright holder. To view a copy of this licence, visit http://creativecommons.org/licenses/by/4.0/. 


\section{References}

1. Allasia, G., Cavoretto, R., De Rossi, A.: A class of spline functions for landmark-based image registration. Math. Methods Appl. Sci. 35, 923-934 (2012)

2. Ambrosio, L., Di Marino, S.: Equivalent definitions of BV space and of total variation on metric measure spaces. J. Funct. Anal. 266(7), 4150-4188 (2014)

3. Angeloni, L.: Approximation results with respect to multidimensional $\varphi$-variation for nonlinear integral operators. Z. Anal. Anwendungen 32(1), 103-128 (2013)

4. Angeloni, L.: A new concept of multidimensional variation in the sense of Riesz and applications to integral operators. Mediterr. J. Math., 14(4), 149 (2017)

5. Angeloni, L., Costarelli, D., Vinti, G.: A characterization of the convergence in variation for the generalized sampling series. Ann. Acad. Sci. Fenn. Math. 43, 755-767 (2018)

6. Angeloni, L., Costarelli, D., Vinti, G.: A characterization of the absolute continuity in terms of convergence in variation for the sampling Kantorovich operators. Medit. J. Math. 16(2), 44 (2019)

7. Angeloni, L., Costarelli, D., Vinti, G.: Convergence in variation for the multidimensional generalized sampling series and applications to smoothing for digital image processing. Ann. Acad. Sci. Fenn. Math. 45, 751-770 (2020)

8. Angeloni, L., Vinti, G.: Convergence in variation and rate of approximation for nonlinear integral operators of convolution type. Results Math. 49, 1-23 (2006)

9. Angeloni, L., Vinti, G.: Convergence and rate of approximation for linear integral operators in $B V^{\varphi}$-spaces in multidimensional setting. J. Math. Anal. Appl. 349, 317-334 (2009)

10. Angeloni, L., Vinti, G.: A sufficient condition for the convergence of a certain modulus of smoothness in multidimensional setting. Comm. Appl. Nonlinear Anal. 20(1), 1-20 (2013)

11. Angeloni, L., Vinti, G.: Convergence and rate of approximation in $B V^{\varphi}\left(\mathbb{R}_{+}^{N}\right)$ for a class of Mellin integral operators. Atti Accad. Naz. Lincei Cl. Sci. Fis. Mat. Natur. 25(3), 217-232 (2014)

12. Angeloni, L., Vinti, G.: Convergence in variation and a characterization of the absolute continuity. Integral Transforms Spec. Funct. 26(10), 829-844 (2015)

13. Appell, J., Banaś, J., Merentes, N.: Bounded variation and around, 17 De Gruyter Series in Nonlinear Analysis and Applications. De Gruyter, Berlin, Germany (2014)

14. Bardaro, C., Butzer, P.L., Stens, R.L., Vinti, G.: Convergence in variation and rates of approximation for Bernstein-type polynomials and singular convolution integrals. Analysis 23, 299-340 (2003)

15. Bardaro, C., Butzer, P.L., Stens, R.L., Vinti, G.: Kantorovich-type generalized sampling series in the setting of Orlicz spaces. Sampling Theory Signal Image Process 6, 29-52 (2007)

16. Bardaro, C., Mantellini, I.: On convergence properties for a class of Kantorovich discrete operators. Numer. Funct. Anal. Optim. 33, 374-396 (2012)

17. Butzer, P.L., Fisher, A., Stens, R.L.: Generalized sampling approximation of multivariate signals: theory and applications. Note di Matematica 10(1), 173-191 (1990)

18. Butzer, P.L., Fisher, A., Stens, R.L.: Generalized sampling approximation of multivariate signals: general theory. Atti Sem. Mat. Fis. Univ. Modena 41(1), 17-37 (1993)

19. Butzer, P.L., Nessel, R.J.: Fourier Analysis and Approximation I. Academic Press, New York-London (1971)

20. Candeloro, D.: L'area di Geöcze, per prodotti di applicazioni, come integrale alla Burkill-Cesari, Istit. Lombardo Accad. Sci. Lett. Rend. A 110(2), 493-513 (1976)

21. Candeloro, D.: The Burkill-Cesari integral and its relation to absolute continuity. Rend. Circ. Mat. Palermo 26(2), 251-274 (1977)

22. Candeloro, D., Di Piazza, L., Musial, K., Sambucini, A.R.: Gauge integrals and selections of weakly compact valued multifunctions. J. Math. Anal. Appl. 441(1), 293-308 (2016)

23. Candeloro, D., Di Piazza, L., Musial, K., Sambucini, A.R.: Relations among gauge and Pettis integrals for cwk(X)-valued multifunctions. Ann. Mat. Pura Appl. 197(4), 171-183 (2018)

24. Coroianu, L., Gal, S.G.: $L^{p}$-approximation by truncated max-product sampling operators of Kantorovichtype based on Féjer kernel. J. Integral Eq. Appl. 29(2), 349-364 (2017)

25. Costarelli, D., Seracini, M., Vinti, G.: A segmentation procedure of the pervious area of the aorta artery from CT images without contrast medium. Math. Methods Appl. Sci. 43, 114-133 (2020)

26. Costarelli, D., Seracini, M., Vinti, G.: A comparison between the sampling Kantorovich algorithm for digital image processing with some interpolation and quasi-interpolation methods. Appl. Math. Comp. 374, 125046 (2020)

27. Costarelli, D., Vinti, G.: Approximation by multivariate generalized sampling Kantorovich operators in the setting of Orlicz spaces, Bollettino U.M.I., Special issue dedicated to Prof. Giovanni Prodi 9(IV), 445-468 (2011) 
28. Costarelli, D., Vinti, G.: An inverse result of approximation by sampling Kantorovich series. Proc. Edinb. Math. Soc. 62(1), 265-280 (2019)

29. Costarelli, D., Vinti, G.: Inverse results of approximation and the saturation order for the sampling Kantorovich series. J. Approx. Theory 242, 64-82 (2019)

30. Costarelli, D., Vinti, G.: Saturation by the Fourier transform method for the sampling Kantorovich series based on bandlimited kernels. Anal. Math. Phys. 9, 2263-2280 (2019)

31. Giusti, E.: Minimal Surfaces and Functions of Bounded Variation, Monographs in Mathematics, vol. 80. Birkhäuser Verlag, Basel (1984)

32. Kivinukk, A., Metsmagi, T.: The variation detracting property of some Shannon sampling series and their derivatives. Sampl. Theory Signal Image Process 13, 189-206 (2014)

33. Orlova, O., Tamberg, G.: On approximation properties of generalized Kantorovich-type sampling operators. J. Approx. Theory 201, 73-86 (2016)

34. Radó, T.: Length and Area, American Mathematical Society Colloquium Publications, vol. 30. American Mathematical Society, New York (1948)

35. Tonelli, L.: Su alcuni concetti dell'analisi moderna. Ann. Scuola Norm. Super. Pisa. 2(11), 107-118 (1942)

36. Unser, M.: Ten good reasons for using spline wavelets. Wavelets Appli Signal Image Process 3169(5), 422-431 (1997)

37. Vinti, C.: Perimetro-variazione. Ann. Scuola Norm. Sup. Pisa 3(18), 201-231 (1964)

38. Vinti, G., Zampogni, L.: A unifying approach to convergence of linear sampling type operators in Orlicz spaces. Adv. Diff. Eq. 16, 573-600 (2011)

Publisher's Note Springer Nature remains neutral with regard to jurisdictional claims in published maps and institutional affiliations.

\section{Affiliations}

\section{Laura Angeloni $^{1} \cdot$ Danilo Costarelli $^{1}$ (D) Marco Seracini ${ }^{1} \cdot$ Gianluca Vinti $^{1}$. Luca Zampogni ${ }^{1}$}

Laura Angeloni

laura.angeloni@unipg.it

Marco Seracini

marco.seracini@dmi.unipg.it

Gianluca Vinti

gianluca.vinti@unipg.it

Luca Zampogni

luca.zampogni@unipg.it

1 Department of Mathematics and Computer Science, University of Perugia, Via Vanvitelli 1, 06123

Perugia, Italy 